\title{
Problemática en la toma de decisiones financieras en las empresas familiares ${ }^{1}$
}

\author{
Paula Andrea Fernández Calle ${ }^{2}$ \\ Verónica Alejandra Guaca Velásquez ${ }^{3}$ \\ Luisa Fernanda Uribe Cardona ${ }^{4}$
}

Recibido: 7 de marzo del 2019

Aprobado: 4 de abril del 2019

Clasificación JEL: M14, D10, D81

1 Fernández, P. A., Guaca, V. A., y Uribe L. F. (2019). Problemática en la toma de decisiones financieras en las empresas familiares. Revista Activos, 16(31), 89-109. DOI: https://doi.org/10.15332/25005278.5317

El escrito hace parte del seminario de investigación de la Especialización en Finanzas de la Universidad Católica Luis Amigó, Medellín, Colombia, asesorado por el profesor Carlos Eduardo Castaño Ríos. El texto hace parte del proyecto de investigación: Diagnóstico contable y financiero a pymes sector alimentos o puntos de venta Universidad Católica Luis Amigó, perteneciente al grupo de investigación Contas, línea de investigación: Contabilidad, ambiente y sociedad, de la Facultad de Ciencias Administrativas, Económicas y Contables, programa de Contaduría Pública.

2 Contadora pública, aspirante al título de especialista en Finanzas de la Universidad Católica Luis Amigó, Medellín, Colombia. Correo electrónico: paulafernandezcontadora@gmail.com

3 Contadora pública, aspirante al título de especialista en Finanzas de la Universidad Católica Luis Amigó, Medellín, Colombia. Correo electrónico: Veronica. guacave@gmail.com

4 Contadora pública, aspirante al título de especialista en Finanzas de la Universidad Católica Luis Amigó, Medellín, Colombia. Correo electrónico: Lufeuca26@hotmail.com 


\title{
Resumen
}

El $87 \%$ de las empresas activas en Antioquia son de índole familiar, aporte significativo a los sectores de la economía nacional. Pero, la ausencia de un protocolo adecuado para tomar decisiones financieras, no les permite alcanzar su madurez económica. Este trabajo comprendió el análisis de los principales obstáculos en el proceso de toma de decisiones financieras a través del estudio de dos casos empresariales, para identificar los factores que inciden en su proceder frente a temas financieros; a través de una metodología cualitativa bajo el método analítico e inductivo. Ambos casos analizados arrojan, en los indicadores de rentabilidad, liquidez y diagnóstico, tendencias bajistas, incluso negativas, que reflejan inadecuadas prácticas de administración en decisiones con criterios subjetivos, conocimiento empírico y satisfacción de necesidades según la oportunidad del negocio, que dificultan el control de los costos y gastos, la conversión de ventas en utilidad y el retorno del capital invertido al inversionista.

Palabras clave: empresa familiar, protocolo de familia, decisiones de inversión, análisis financiero.

\section{Problems in making financial decisions in family businesses}

\begin{abstract}
$87 \%$ of the companies active in Antioquia are of a family nature, a significant contribution to the sectors of the national economy. But, the absence of an adequate protocol to make financial decisions, does not allow them to reach their economic maturity. This work includes the analysis of the main obstacles in the financial decision-making process through the study of two business cases, to identify the factors that influence their actions in relation to financial issues; through a qualitative methodology under the
\end{abstract}


Analytical and inductive method. Both analyzed cases show, in the indicators of Profitability, Liquidity and Diagnosis, bearish tendencies, even negative ones, that reflect inadequate management practices in decisions with subjective criteria, empirical knowledge and satisfaction of needs according to the business opportunity that hinder the control of costs and expenses, the conversion of sales into profits and the return of the capital invested to the investor.

Keywords: Family business, family protocol, investment decisions, financial analysis.

\section{Introducción}

Los constantes cambios de la economía mundial exigen que las compañías implementen estrategias que se traduzcan en ventajas administrativas y comerciales competitivas que contribuyan a consolidarse en el mercado, permanecer a través del tiempo y generar valor agregado a sus grupos de interés. Dichas estrategias deben elaborarse considerando varios factores como una planeación estratégica clara y definida, una adecuada toma de decisiones financieras basadas en información oportuna y confiable, y una gestión administrativa, contable y operativa formal que contribuya al logro de los objetivos organizacionales. Lo anterior representa un reto mayor para las empresas mipymes de tipo familiar que en ocasiones se constituyen a partir de la informalidad y orientan de esta forma los procesos dentro de sus compañías.

Por lo anterior, el presente trabajo comprendió el estudio de los principales obstáculos en el proceso de toma de decisiones financieras de dos compañías seleccionadas como muestras de empresas familiares mipymes ubicadas en la ciudad de Medellín, con el fin de identificar en ellas los principales factores que inciden en la determinación de su proceder frente a temas netamente financieros. 
Hay diversas definiciones sobre lo que es una empresa familiar, para tal fin se pueden tomar en cuenta varios aspectos, como el poder de intervención de familiares en la dirección y toma de decisiones. Para este estudio se define como empresa familiar a aquella en la que los miembros de una familia ejercen control y administran teniendo la expectativa de que la compañía permanecerá en el tiempo; por lo que el concepto general de empresa familiar no está ligado necesariamente al tamaño de la misma o a un sector en especial (Romero, 2006).

\section{Marco de referencia}

De acuerdo a lo dispuesto en la Guía Colombiana de Gobierno Corporativo para Sociedades Cerradas y de Familia (Confecámaras, 2009), se deben establecer unos lineamientos para que las empresas familiares puedan llevar a cabo su determinada labor. Para tal fin, según el documento mencionado, es de vital importancia regular el ámbito en que interactúan la empresa y la familia con el fin de que se sorteen de manera exitosa los obstáculos inherentes a este tipo de organizaciones, todo esto encaminado a aumentar la riqueza del empresario y el fortalecimiento de la generación de empleo y la correcta toma de decisiones.

El impacto que tienen las empresas familiares sobre la economía del país es bastante significativo, ya que según los resultados arrojados por la Encuesta Nacional de Gobierno Corporativo, de un total de 7414 compañías que dieron respuesta a la consulta, el $53.70 \%$ corresponde a empresas familiares y aun teniendo en cuenta que la encuesta no fue resuelta por la totalidad de empresas micro y medianas; se puede concluir entonces que el porcentaje que compone este tipo de empresas en la economía del país, supera el $70 \%$. No obstante, aunque gran parte de las empresas familiares están en las pymes, también hay porcentajes importantes relacionados con las grandes empresas del país, pues el $14 \%$ de las empresas familiares en Colombia son las principales grandes empresas. Este dato es altamente confiable dada la obligatoriedad que tienen estas de responder las principales encuestas que las miden. 
Siguiendo en la línea de las cifras otorgadas por Confecámaras, de acuerdo con diferentes estudios realizados, el $40 \%$ de las empresas reportadas en su Formulario de Prácticas Empresariales son empresas familiares. Por esto y por el hecho de que estas empresas componen el $60 \%$ del PIB a nivel Latinoamérica, se deben mirar muy de cerca sus diferentes problemáticas para poder tomar medidas que propendan en su mejoramiento y permanencia en el tiempo.

Por otro lado, Confecáramas toma como base lo indicado por la Superintendencia de Sociedades como resultado de la encuesta de Empresas de Familia y cálculos del Grupo de conglomerados realizados en el año 2004, que relata que los principales conflictos que enfrenta una empresa familiar son la poca preparación que tienen para enfrentar el cambio generacional y la combinación de decisiones familiares. No obstante lo anterior, hay otras circunstancias que juegan adversamente en las empresas familiares, tales como la concentración del poder en el fundador de la misma y los conflictos familiares, que pueden llevar en ocasiones a decisiones precipitadas, ausencia de procedimientos y dificultades financieras, entre otras.

Lo anterior permite argüir que es de suma importancia promover en las empresas familiares prácticas adecuadas de gobierno corporativo aboliendo las viejas tendencias de ensayo y error en donde se pone en riesgo no solo la dilapidación de la riqueza patrimonial de la sociedad como tal, sino también, el crecimiento y fortalecimiento de la economía del país. Prueba de esto está en que cada vez son más los empresarios que acuden a acompañamientos en materia administrativa más profesional y al fortalecimiento de sus juntas directivas (Gil y Giner, 2013).

En tal medida es pertinente entonces crear protocolos de familia e instituciones familiares como lo son la Asamblea de Familia, que es el órgano que rige las relaciones familiares; sus funciones principales son crear espacios benéficos con objetivos claros como formar una familia más fuerte y a la vez un negocio más fuerte entre otras; y el Consejo de Familia cuyas funciones entre varias sería armonizar las relaciones entre la familia y la 
empresa con el fin de que las decisiones que afecten de una u otra forma a la familia no incidan en los resultados que se puedan observar en los estados financieros de la empresa, y a su vez que las principales decisiones de la empresa que se tomen encaminadas al fortalecimiento de la sociedad no afecten a ningún miembro de la familia. La implementación de estos órganos en cada empresa familiar, dependerá finalmente de las necesidades particulares que cada una tenga (González, Guzmán y Trujillo, 2010).

Otra de las funciones importantes del Consejo de Familia es establecer un procedimiento para la presentación de candidatos idóneos al plan de sucesión del ejecutivo principal. A nivel mundial sólo el $30 \%$ de las empresas familiares superan el tránsito de la primera y segunda generación y sólo entre el $5 \%$ y el $15 \%$ llega a la tercera generación. En el caso colombiano un $75.8 \%$ de las empresas está en manos de la primera generación dejando un porcentaje mínimo a las que llegaron a segunda y tercera generación.

La realidad de la empresa familiar suele tornarse compleja ya que los miembros de la familia deben asumir roles de socios y gerentes lo cual lleva a presentar un alto grado de vulnerabilidad ante conflictos que se generen a causa de problemas intrafamiliares, demostrando que el importante proceso de toma de decisiones se lleva de manera muy informal y con plena falta de objetividad y juicio lo que a la larga conduce a consecuencias negativas para la empresa. Estas decisiones, si bien es cierto pueden ser a nivel organizacional y administrativo, claramente en su mayoría afectan la parte financiera de la empresa en el sentido en que para los empresarios es muy difícil separar sus responsabilidades de índole familiar a las netamente empresariales (Quejada y Ávila, 2016).

Teniendo en cuenta lo anterior, y ante una evidente necesidad de orientación, no es posible decir que en la actualidad exista como tal una reglamentación o normatividad que guíe el buen proceder de las empresas familiares en el ámbito nacional. Sin embargo, a falta de ello, es posible encontrar tanto en el país como internacionalmente un buen número de literatura relacionada, siendo válido resaltar para efectos de la presente investigación informes y conceptos que llevan a concluir que un factor 
predominante en el declive de las empresas familiares es la ineficiencia en la toma de decisiones asertivas para la empresa.

Con relación a esto, se han abordado en la literatura diversas problemáticas que afrontan las empresas familiares como la inexistencia de planes estratégicos (Beltrán, Torres, Camargo y Pérez, 2004); informalidad en el proceso de toma de decisiones (Beltrán, 2006); la toma de decisiones como una técnica inherente a la administración de empresas (Castillo Hernández, 2006); la dirección y sucesión de una empresa cuya propiedad está en manos de uno o más miembros de un grupo familiar, tendrá influencia familiar (Navarrete, 2008); el clima organizacional inadecuado que reduce las posibilidades de crecimiento (Sandoval y Guerrero, 2010); decisiones de inversión, financieras y de administración de activos (Van Home y Wachowicz, 2002) y el nivel de escolaridad de los directivos de la empresa familiar que incide en la forma como se evalúan proyectos de inversión (Vecino, Rojas y Muñoz, 2015).

\section{Metodología}

La investigación desarrollada tiene un enfoque cualitativo, ya que se dirige a interpretar la forma y razón de la toma de decisiones de las empresas familiares en la actualidad; mediante el análisis descriptivo y desde el paradigma interpretativo, el cual se encarga de estudiar una situación en el contexto en que ocurre, tratando de comprender una realidad.

Se utilizó un tipo de investigación descriptivo y analítico, puesto que narra el contexto del proceder decisorio de las empresas familiares, examinando factores en común y teniendo presente, incluso, aquellos que en un principio se muestren poco sobresalientes.

En este orden de ideas, se realizó un estudio multicaso con el que se pretendía indagar un fenómeno, población o condición general a través de una lista de preguntas semiestructurada, cuyos resultados fueron analizados, interpretados y posteriormente descritos en un informe. Los casos o 
situaciones evaluados parten de concepciones generalizadas en el ambiente organizacional sobre la toma de decisiones económicas en las empresas familiares, seleccionadas por la participación en su administración a través del ejercicio profesional contable.

Se llevó a cabo un método inductivo, ya que se efectuó una completa identificación de las partes involucradas en la toma de decisiones en una mipyme que concluye en una descripción de los principales problemas en el proceso de inversión y de decisión económica.

Para efectos de veracidad de la información, se estudiaron dos escenarios económicos dentro de las empresas familiares como referentes, una perteneciente al sector comercial y un segundo caso del sector productivo. Para tal fin se tomó como período de análisis el comportamiento financiero de ambas empresas durante los últimos tres años estudiando los rubros más significativos de sus estados financieros y que a su vez muestren una variación sustancial en los resultados de cada organización; adicionalmente se analizó el entorno empresarial y familiar que llevó a la gerencia a tomar ciertas decisiones y las implicaciones económicas que acarrearon.

Para la recolección de los datos necesarios, se realizó una entrevista oral a los administradores de las empresas seleccionadas, lo cual constituye la fuente primaria de la investigación (ver Anexo 1).

\section{Resultados}

Las entrevistas fueron aplicadas a dos empresas familiares, en un primer caso a cargo de los propietarios (caso 1) y un segundo caso aplicado por un empleado perteneciente al área financiera de la empresa (caso 2). Esto permitió evidenciar la percepción de los primeros y el juicio profesional del segundo sobre la problemática de la toma de decisiones financieras en empresas familiares.

En el caso 1 se aplicaron dos entrevistas a familiares administrativos de la empresa, hallando que ambos comparten una visión relativa y poco 
estructurada de la realidad de la compañía, ya que manifiestan no encontrar obstáculo ni limitante alguno al compartir decisiones con otros familiares, bajo un sustento no financiero sino únicamente personal, afirmando que la viabilidad comercial está garantizada puesto que en conjunto trabajan para lograr un objetivo en común, es decir, que su crecimiento no depende de su conformación sino de la proyección que tienen. En contraste a su tesis, ambos reconocen que la toma de decisiones que implican manejo de dinero la han realizado hasta la fecha con base a razones financieras no analizadas sino en las que se presentan en el día a día de su actividad comercial. Adicionalmente, operan sin la existencia de un plan estratégico, enfocándose únicamente en controlar los ingresos de efectivo que se dan, pero con una inestable estructura de distribución de los mismos.

Adicionalmente, los administrativos definen como único obstáculo empresarial, el mantener un equilibrio (no económicamente hablando) entre los espacios familiares y los laborales.

Por último, indican no haber realizado inversiones propias a la fecha con recursos de la compañía, sin embargo, aceptan que actualmente no hay una independencia absoluta de los gastos de ambas partes, ya que no se tiene establecido un salario, sino que se dispone del efectivo según la necesidad y oportunidad.

Para el caso 1, los entrevistados concluyen que los familiares que hacen parte de la empresa no están ejerciendo su rol por interés propio, sino que son los idóneos para el cargo, sin embargo, se estima una falencia en dicha afirmación puesto que ya se cuenta con un antecedente de quiebra por conflictos familiares y aun así no se estableció en esta segunda etapa de operación un protocolo que limite y controle las decisiones e intervenciones de los involucrados.

En el caso 2, se encuentra una visión más objetiva de la situación de la compañía, ya que la entrevista fue aplicada a un empleado del área financiera, que se encuentra inmerso y completamente relacionado con los resultados de la empresa y que, si bien participa desde un rol de asesoría, 
se encuentra limitado ante respuestas y actos improvisados que no obedecen a las sugerencias dadas. Un caso puntual de lo antes mencionado es al momento de lanzar un nuevo producto al mercado, este proceso se hace sin evaluar acertadamente los costos para poder estipular correctamente el precio de venta, situación que a la larga termina siendo totalmente desfavorable para los resultados de la operación. Situaciones como estas se presentan en la empresa en todas sus áreas.

Se logró evidenciar que el hecho de que la empresa sea familiar, afecta en gran medida a las decisiones que se tomen ya que hay un marcado impacto de conflicto de intereses en donde se le da prioridad incluso a ciertos miembros de la familia, aunque estén en el mismo rango jerárquico.

El empleado entrevistado destaca que la empresa tiene un comportamiento organizado con relación a los recursos familiares y los de la empresa, aunque se dan situaciones en las que se utilizan para fines personales, la gerencia tiene claro que las dos cosas no se deben mezclar y bajo ese criterio son tomadas este tipo de decisiones, evidencia de ello es el avance que se ha tenido al acudir hoy en día a los estados financieros, situación que no se daba años atrás, y de lo que se espera poco a poco represente una fuente única para la toma de decisiones.

En este proceso, se identificaron tres grandes categorías que definen el actuar de los directivos de las empresas familiares analizadas al momento de decidir financieramente: decisiones con criterios subjetivos, decisiones basadas en conocimiento empírico y decisiones basadas según la necesidad, contingencia u oportunidad. Lo anterior se resume en la Figura 1.

En cuanto al análisis de la información financiera mediante el uso de indicadores de rentabilidad, liquidez y de diagnóstico para los períodos 2015, 2016 y 2017, se encontraron los siguientes hallazgos, resumidos en las Tablas 1 y 2. 
Figura 1. Necesidades percibidas

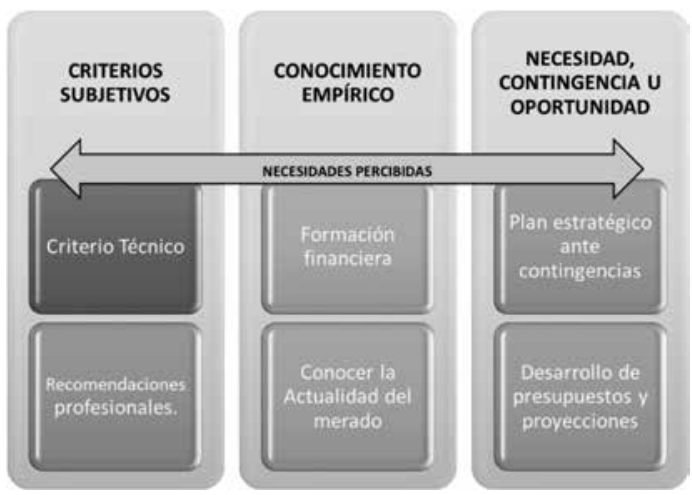

Fuente: elaboración propia (2018).

Tabla 1. Indicadores financieros caso 1

\begin{tabular}{|c|c|c|c|c|c|}
\hline & \multirow{2}{*}{\multicolumn{2}{|c|}{ INDICADORES FINANCIEROS }} & \multicolumn{3}{|c|}{ CASO 1} \\
\hline & & & 2017 & 2016 & 2015 \\
\hline \multirow{11}{*}{ 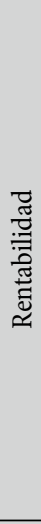 } & $\begin{array}{c}\text { Rentabilidad neta } \\
\text { del activo (Du } \\
\text { Pont) }= \\
\end{array}$ & $\begin{array}{c}\text { (Utilidad Neta/Ventas) })^{*}(\text { Ventas/ } \\
\text { Activo Total) }\end{array}$ & $2,13 \%$ & $-1,51 \%$ & $-25,59 \%$ \\
\hline & \multirow{2}{*}{ Margen Bruto $=$} & Ventas netas - Costos de ventas & \multirow{2}{*}{$24,17 \%$} & \multirow{2}{*}{$22,53 \%$} & \multirow{2}{*}{$21,36 \%$} \\
\hline & & Ventas & & & \\
\hline & \multirow{2}{*}{$\begin{array}{c}\text { Margen Operacional } \\
=\end{array}$} & Utilidad Operacional & \multirow{2}{*}{$1,41 \%$} & \multirow{2}{*}{$0,97 \%$} & \multirow{2}{*}{$-4,00 \%$} \\
\hline & & Ventas & & & \\
\hline & \multirow{2}{*}{$\begin{array}{l}\text { Margen Neto de } \\
\text { Utilidad }\end{array}$} & Utilidad Neta & \multirow{2}{*}{$0,50 \%$} & \multirow{2}{*}{$-0,23 \%$} & \multirow{2}{*}{$-4,48 \%$} \\
\hline & & Ventas & & & \\
\hline & \multirow{2}{*}{$\begin{array}{l}\text { ROE Rendimiento } \\
\text { Patrimonio }\end{array}$} & Utilidad Neta & \multirow{2}{*}{$9,62 \%$} & \multirow{2}{*}{$-6,63 \%$} & \multirow{2}{*}{$-96,89 \%$} \\
\hline & & Patrimonio & & & \\
\hline & \multirow{2}{*}{$\begin{array}{l}\text { Rentabilidad neta } \\
\text { del activo ROA = }\end{array}$} & Utilidad Neta & \multirow{2}{*}{$2,13 \%$} & \multirow{2}{*}{$-1,51 \%$} & \multirow{2}{*}{$-25,59 \%$} \\
\hline & & Total Activo & & & \\
\hline \multirow{4}{*}{ 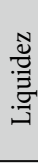 } & \multirow{2}{*}{$\begin{array}{l}\text { Razón circulante o } \\
\text { corriente }=\end{array}$} & Activo Corriente & \multirow{2}{*}{1,27} & \multirow{2}{*}{1,23} & \multirow{2}{*}{1,24} \\
\hline & & Pasivo Corriente & & & \\
\hline & \multirow{2}{*}{$\begin{array}{l}\text { Razón de la prueba } \\
\text { ácida }\end{array}$} & Activo Corriente - Inventarios & \multirow{2}{*}{0,54} & \multirow{2}{*}{0,56} & \multirow{2}{*}{0,91} \\
\hline & & Pasivo Corriente & & & \\
\hline \multirow{4}{*}{ 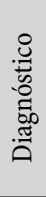 } & EBITDA= & $\begin{array}{c}\text { UO + DEPRECIACIÓN + } \\
\text { AMORTIZACIÓN + PROVISIONES }\end{array}$ & 11.670 .821 & 9.200 & -30.568 \\
\hline & PUNTO DE & Costos fijos & \multirow{2}{*}{53,66} & \multirow{2}{*}{80,07} & \multirow{2}{*}{$-19,65$} \\
\hline & EQUILIBRIO = & Márgen de Contribución & & & \\
\hline & $\mathrm{KNTO}=$ & $\mathrm{CxC}+$ Inventarios - $\mathrm{CxP}$ Proveedores & 8.318 .333 & -33.533 & -65.321 \\
\hline
\end{tabular}

Fuente: elaboración propia (2018). 
El margen bruto para el caso 1 permite inferir que la empresa ha venido aumentando la rentabilidad de las ventas frente a su costo para cubrir óptimamente los gastos operativos y generar utilidad. Lo anterior se revierte con el margen operacional dado que se tiene un resultado negativo en el año 2015 y un margen muy estrecho en los dos años posteriores, lo que indica que sus elevados gastos operacionales de administración y ventas hacen que el negocio sea poco lucrativo en sí mismo, independientemente de la forma como ha sido financiado. El margen neto confirma los resultados anteriores al arrojar cifras negativas para los dos primeros períodos analizados, mostrando la pérdida de la empresa por cada unidad de venta. Así mismo, es muy importante destacar que la rentabilidad operacional del patrimonio que es aquella que se ofrece a los socios o accionistas por el capital invertido, generó perdida los dos primeros años y presentó una leve recuperación en el último período.

La liquidez, definida como la capacidad que tiene una empresa para generar fondos suficientes para cubrir sus obligaciones a corto plazo, refleja un nivel razonable en la empresa del caso 1 puesto que con los activos disponibles tiene para cubrir más de una vez los pasivos contraídos en el corto plazo. Esta cobertura se logra en mayor medida por los activos diferentes al inventario como lo refleja la razón de la prueba ácida, lo que indica que la empresa del caso 1 cuenta con un alto índice de liquidez inmediata, ya que los inventarios son más lentos para convertir en efectivo.

Para el caso 1 se tiene un EBITDA positivo en los dos últimos años, lo que refleja que la capacidad de la empresa para generar beneficios económicos considerando únicamente su actividad productiva va en aumento. Pese a esto, la utilidad generada por la operación se disminuye notablemente por la carga del nivel de endeudamiento de la empresa.

Como se puede observar en la Tabla 2, la empresa del caso 2 tiene un buen margen bruto, esto indica que ha mantenido una buena política para establecer los precios de sus productos y mantiene una relación óptima entre las ventas y los costos; sin embargo, el indicador tiene una disminución un tanto significativa con relación al año 2015 lo que podría 
Tabla 2. Indicadores financieros caso 2

\begin{tabular}{|c|c|c|c|c|c|}
\hline & \multirow{2}{*}{\multicolumn{2}{|c|}{ INDICADORES FINANCIEROS }} & \multicolumn{3}{|c|}{ CASO 1} \\
\hline & & & 2017 & 2016 & 2015 \\
\hline \multirow{11}{*}{ 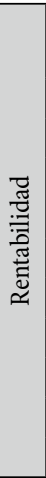 } & $\begin{array}{l}\text { Rentabilidad neta del } \\
\text { activo (Du Pont) }=\end{array}$ & $\begin{array}{c}\text { (Utilidad Neta/Ventas) }^{*} \\
\text { (Ventas/Activo Total) }\end{array}$ & $-1,30 \%$ & $1,94 \%$ & $2,32 \%$ \\
\hline & \multirow{2}{*}{ Margen Bruto $=$} & Ventas netas - Costos de ventas & \multirow{2}{*}{$66,36 \%$} & \multirow{2}{*}{$68,27 \%$} & \multirow{2}{*}{$59,74 \%$} \\
\hline & & Ventas & & & \\
\hline & \multirow{2}{*}{ Margen Operacional = } & Utilidad Operacional & \multirow{2}{*}{$8,77 \%$} & \multirow{2}{*}{$7,07 \%$} & \multirow{2}{*}{$7,92 \%$} \\
\hline & & Ventas & & & \\
\hline & \multirow{2}{*}{ Margen Neto de Utilidad } & Utilidad Neta & \multirow{2}{*}{$-0,84 \%$} & \multirow{2}{*}{$0,75 \%$} & \multirow{2}{*}{$0,92 \%$} \\
\hline & & Ventas & & & \\
\hline & \multirow{2}{*}{$\begin{array}{l}\text { ROE Rendimiento } \\
\text { Patrimonio }\end{array}$} & Utilidad Neta & \multirow{2}{*}{$-19,45 \%$} & \multirow{2}{*}{$12,25 \%$} & \multirow{2}{*}{$10,68 \%$} \\
\hline & & Patrimonio & & & \\
\hline & \multirow{2}{*}{$\begin{array}{l}\text { Rentabilidad neta del } \\
\text { activo ROA = }\end{array}$} & Utilidad Neta & \multirow{2}{*}{$-1,30 \%$} & \multirow{2}{*}{$1,94 \%$} & \multirow{2}{*}{$2,32 \%$} \\
\hline & & Total Activo & & & \\
\hline \multirow{4}{*}{ 胥 } & \multirow{2}{*}{$\begin{array}{l}\text { Razón circulante o } \\
\text { corriente }=\end{array}$} & Activo Corriente & \multirow{2}{*}{1,74} & \multirow{2}{*}{1,41} & \multirow{2}{*}{1,16} \\
\hline & & Pasivo Corriente & & & \\
\hline & \multirow{2}{*}{ Razón de la prueba ácida } & Activo Corriente - Inventarios & \multirow{2}{*}{1,13} & \multirow{2}{*}{1,18} & \multirow{2}{*}{1,03} \\
\hline & & Pasivo Corriente & & & \\
\hline \multirow{3}{*}{ 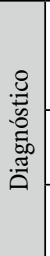 } & EBITDA= & $\begin{array}{c}\text { UO + DEPRECIACIÓN } \\
+ \text { AMORTIZACIÓN + } \\
\text { PROVISIONES } \\
\end{array}$ & 164.123 .119 & 166.697.348 & 143.617.235 \\
\hline & $\begin{array}{c}\text { PUNTO DE EQUILIBRIO } \\
=\end{array}$ & Costos fijos & 3,84 & 4,49 & 5,08 \\
\hline & $\mathrm{KNTO}=$ & $\begin{array}{c}\mathrm{CxC}+\text { Inventarios - } \mathrm{CxP} \\
\text { Proveedores }\end{array}$ & 265.945 .467 & 80.531 .540 & 62.044 .578 \\
\hline
\end{tabular}

Fuente: elaboración propia.

tomarse como una señal para tener en cuenta en los períodos futuros que se analicen de la compañía. En lo que concierne al margen operacional, es quizá donde se empiezan a ver los problemas de la empresa ya que tiene un margen muy estrecho, que, si bien tiene un pequeño crecimiento para el año 2017, no es suficiente ya que la empresa incurre en el pago de salarios elevados a personal familiar que no representa ciertamente un aporte para el crecimiento en ventas de la empresa. Infiriendo que la empresa puede ser rentable operativamente, aun con sus márgenes estrechos, cuando se analiza el margen neto de utilidad, el panorama se vuelve más oscuro para esta empresa ya que para el año 2017 el porcentaje resultante es negativo; 
esto se da por el aumento del nivel de endeudamiento que hace encarecer la carga financiera de la empresa absorbiendo casi por completo su utilidad.

Por su parte, los indicadores de liquidez del caso 2 presentan un escenario positivo ya que los activos corrientes cubren eficientemente los pasivos a corto plazo en que la empresa incurre. Este indicador ha aumentado en los años de estudio y aunque los resultados son positivos, al compararlo con los resultados de los indicadores de rentabilidad, se puede concluir que el porcentaje de pasivo a corto plazo es muy bajo frente a la deuda total. Sin embargo, al aplicar la prueba ácida al indicador se tiene la capacidad de pago eficiente del pasivo a corto plazo.

Los indicadores de diagnóstico de la empresa del caso 2 muestran un EBITDA que va en aumento, esto quiere decir que la empresa tiene una buena capacidad para generar caja y que operativamente tiene buenos resultados, sin embargo, se debe reiterar que, en cierta medida, el resultado de la empresa del caso 2 se ve afectado por la carga financiera a través del endeudamiento a largo plazo.

En cuanto al capital de trabajo neto, el estudio muestra un incremento muy significativo en los niveles de capital de trabajo necesarios para poder operar y en ese sentido un debilitamiento en la facilidad para volver efectivo el activo, lo que puede ser una respuesta a la poca liquidez que tiene la empresa y su necesidad de buscar recursos vía endeudamiento financiero.

\section{Conclusiones}

Los resultados financieros arrojados por los indicadores aplicados y las entrevistas realizadas a los propietarios (caso 1) y al empleado del área financiera (caso 2), infieren que la dirección de estas compañías tienden a ejecutar decisiones que tienen un efecto nocivo en los resultados a corto y mediano plazo como lo muestran los bajos márgenes (incluso negativos) de la utilidad operativa, el margen neto, el rendimiento del patrimonio y en el EBITDA, cuyos indicadores denotan la ineficiencia de la administración de 
ambas empresas para controlar los costos y gastos, y así convertir las ventas en utilidad y retornar el capital invertido al inversionista o propietario.

Según lo observado, puntualmente en el análisis del caso 1, los gestores de las empresas familiares en muchas ocasiones no son conscientes de las malas decisiones que se toman aduciendo que para ellos todo está en orden y marcha bien. Para la empresa del caso 2, aunque el gestor es consciente de que el negocio no marcha como debe ser, sigue siendo más valedera su opinión frente a las diversas situaciones decisivas de la empresa.

El panorama anterior muestra la necesidad que tienen las empresas familiares, sobre todo las pymes, de establecer protocolos para el buen manejo de los diversos asuntos relacionados con la familia, ya que se evidencia que en muchos casos los trabajadores que hacen parte de la familia, ocupan cargos con salarios onerosos y poco representativos para el cumplimiento de los objetivos trazados.

De acuerdo a lo anterior, se puede determinar que se hace totalmente necesario el nombramiento de una junta directiva que se haga cargo de tomar las decisiones que puedan tener una incidencia significativa en la empresa y que esté conformada por lo menos por una persona ajena al núcleo familiar, para que se satisfagan las necesidades que surjan en las diferentes etapas empresariales y que se suplan las decisiones con criterios subjetivos, con conocimiento empírico, por contingencia u oportunidad por recomendaciones de profesionales con criterio técnico; formación financiera que establezca una relación con el dinero para generar valor a través de la planificación y el aprovechamiento de oportunidades y, por la elaboración y ejecución de presupuestos que permitan estimar y administrar los recursos en pro del cumplimiento de los objetivos empresariales.

Por último, la experiencia obtenida en las empresas de los dos casos estudiados, refleja la poca participación que tienen las recomendaciones impartidas por el contador y la poca o nula importancia que en muchos de los casos tienen los estados financieros para la acertada toma de decisiones, lo cual refleja resultados poco prometedores con el paso del tiempo. 


\section{Referencias}

Beltrán, A. (2006). Los 20 problemas de la pequeña y mediana industria. Sotavento M.B.A., (7), 8-15. Recuperado de https://dialnet.unirioja.es/descarga/articulo/5137544.pdf

Beltrán, A., Torres, E., Camargo, R., y Pérez, C. B. (2004). Pymes, un reto a la competitividad. Bogotá D. C., Colombia: Editorial Universidad Externado de Colombia.

Cámara de Comercio de Medellín para Antioquia. (2018). Estructura empresarial 2017. Medellín, Colombia. Recuperado de http://www.camaramedellin.com. co/site/Servicios-Empresariales/Informacion-Empresarial/EstadisticasCamara.aspx

Castillo Hernández, M. (2006). Toma de decisiones en las empresas: Entre el arte y la técnica. Bogotá D. C., Colombia: Ediciones Uniandes. DOI: 9586956725 , 9789586956727

Cibrán Ferraz, P., Prado Román, C., Crespo Cibrán, C., Huarte, M. A., y Galbán, C. (2013). Planificación Financiera. Madrid, España: ESIC Editorial.

Confecámaras. (2009). Guía Colombiana de Gobierno Corporativo para Sociedades Cerradas y de Familia. Bogotá D. C., Colombia: ConfecámarasSupersociedades-Cámara de Comercio de Bogotá. DOI: 978-958-688-283-5

Gil Estallo, M., y Giner de la Fuente, F. (2013). Cómo crear y hacer funcionar una empresa. Madrid, España: ESIC Editorial. DOI: 8473569709, 9788473569705

Quejada Pérez, F., y Ávila Gutiérrez, J. (2016). Empresas familiares: Conceptos, teorías y estructuras. Recuperado de http://www.scielo.org.co/pdf/ean/n81/ n81a08.pdf

González Ferrero, M., Guzmán Vásquez, A., y Trujillo Dávila, M. A. (Enero de 2010). The role of heirs in family businesses: The case of Carvajal. Recuperado de http://www.scielo.org.co/scielo. php?pid=S0121-50512010000100005\&script=sci_arttext\&tlng=en

Navarrete, M. (2008). Transición generacional en las empresas familiares mexicanas fabricantes de pinturas y tintas. Premio UDEM-Adalberto Viesca Sada, n. ${ }^{\circ}$ 16, Centro de Empresas Familiares de la Universidad de Monterrey, México.

Romero, L. E. (2006). Competitividad y productividad en empresas familiares pymes. Revista EAN(57), 131-142. Recuperado de http://www.redalyc.org/ pdf/206/20605708.pdf 
Sandoval, J. H., y Guerrero, D. E. (2010). Empresas familiares en Colombia: Hacia la construcción de un modelo de gestión comercial. U.D.C.A Actualidad \& Divulgación Científica, 13(1), 135-146. Recuperado de http://www.scielo.org. co/pdf/rudca/v13n1/v13n1a15.pdf

Van Horne, J. C., y Wachowicz, J. M. (2002). Fundamentos de administración financiera. México D. F., México: Pearson Educación de México S. A. de C. V. Vecino, C. E., Rojas, S. C., y Muñoz, Y. (Enero de 2015). Prácticas de evaluación financiera de inversiones en Colombia. Estudios Gerenciales 31, 41-49. Recuperado de https://reader.elsevier.com/reader/sd/pii/S01235923140016 12?token=649BD0F504ADCA71EB35E448E513481B45666233198CB5D1B E26492D592B084D586814F78644F5B03B9C3EC7CEF06BD0 


\section{ANEXO 1}

1. ¿A qué sector económico pertenece su empresa?
a. Agricultura
b. Comercio
c. Industria
d. Servicios

2. ¿Cuánto tiempo tiene de funcionar su empresa?
a. 1 a 3 años
b. 3 a 9 años
c. 9 a 19 años
d. Más de 19 años

3. ¿Cuántos empleados tiene su empresa?
a. De 1 a 5 empleados
b. De 6 a 30 empleados
c. De 31 a 100 empleados
d. Más de 100 empleados

4. ¿Es su empresa una empresa familiar?
a. $\mathrm{Si}$
b. No

5. Si la anterior pregunta es afirmativa indique:

¿Cuántos accionistas familiares tiene actualmente la empresa?
a. Uno
b. Entre dos y cinco
c. Entre seis y veinte
d. Más de veinte 
6. ¿Cree usted que el hecho de que la propiedad sea familiar limita las posibilidades de crecimiento de la empresa?
a. $\mathrm{Si}$
b. No ¿Por qué?

7. De los miembros de la familia que son accionistas, ¿cuántos trabajan en la empresa?
a. Todos
b. Algunos
c. Ninguno

8. ¿Cuáles de las siguientes herramientas financieras utilizan en su empresa? Puede seleccionar más de una opción.
a. Estados financieros
b. Estado de flujo de efectivo
c. Razones financieras
d. Ventas proyectadas
e. Presupuesto
f. Costo de oportunidad

9. ¿Existe un plan estratégico a mediano o largo plazo?
a. $\mathrm{Si}$
b. No

10. En relación al manejo de efectivo, ¿con cuál de las siguientes opciones se identifica?
a. Separa el dinero personal del dinero del negocio
b. Tiene un salario establecido para sus labores realizadas en el negocio
c. Controla los ingresos de efectivo
d. Controla los egresos o salidas de efectivo 
11. ¿Cómo considera que está la situación actual de su empresa, comparándola con los últimos dos años? En términos generales, ¿diría usted que está mucho mejor... mejor... peor... o mucho peor?
a. Mucho mejor
b. Mejor
c. Igual
d. Peor
e. Mucho peor

12. Al momento de tomar decisiones financieras, ¿quién participa en el proceso?
a. Junta Directiva
b. Gerencia en cooperación con el área financiera
c. Familiares influyentes en las áreas responsables
d. Las decisiones son tomadas sin un orden establecido, solo siguiendo la necesidad del momento

13. ¿Cuáles cree usted que son los principales obstáculos y desafíos a los que se enfrentan las pymes familiares?

14. ¿Durante el tiempo de operación de la empresa se ha encontrado ante la oportunidad de destinar recursos de la misma para fines personales o para lucro de algún miembro de la familia?
a. $\mathrm{Si}$
b. No

Si su respuesta es afirmativa indique algunos ejemplos

15. Al momento de invertir los recursos de la empresa para fines personales, ¿ toma en cuenta algún informe financiero para saber en qué estado se encuentra la empresa?
a. $\mathrm{Si}$
b. No
¿Por qué? 
16. ¿Toma en consideración las recomendaciones que le da el financiero o contador de la empresa en el momento de destinar recursos de la empresa a fines personales?
a. $\mathrm{Si}$
b. No

¿Por qué?

17. ¿Considera que las decisiones que se toman con relación a los recursos de la compañía se toman de forma sensata que no esté movida por un conflicto de intereses?

a. $\mathrm{Si}$

b. No

\section{Explique}

18. Teniendo en cuenta que el principal activo que tiene una empresa es el recurso humano, al momento de nombrar o promover a un colaborador, ise le da prelación a los miembros de la familia así estos estén poco capacitados para el desempeño del mismo?
a. $\mathrm{Si}$
b. No
¿Por qué? 